\title{
Endocrine evaluation of patients after brain injury: what else is needed to define specific clinical recommendations?
}

\author{
Gemma Sesmilo, Irene Halperin, Manuel Puig-Domingo \\ Servei d'Endocrinologia, Hospital Clínic, Universitat de Barcelona, Barcelona, Spain
}

\section{INTRODUCTION}

Brain injury (BI), both head trauma (TBI) and subarachnoid hemorrage (SAH), has consistently been recognized as an important cause of pituitary dysfunction. ${ }^{1}$ Different studies have estimated that $20-50 \%$ of patients who have suffered BI will develop some degree of hypopituitarism..$^{1-9}$ The high incidence of BI ranks it as potentially the most important cause of hypothalamic-pituitary dysfunction. Although many efforts have been made to explore and define the management of BI patients from the endocrine standpoint, some important questions still remain.

In 2005, a group of experts drew up a Consensus Statement, which included a review of the knowledge on BI induced hypopituitarism and proposed different measures to increase awareness of the problem. ${ }^{10}$ General recommendations regarding management of patients in this setting were put forward. Recently, Agha and Thompson ${ }^{3}$ carefully reviewed the new evidence in the field and offered clinical recommendations, although no specific protocol was defined ${ }^{3}$.

Key words: Brain injury, Growth hormone deficiency, Hypogonadism, Hypopituitarism

Address for correspondence:

Gemma Sesmilo, Servei d'Endocrinologia, Hospital Clínic, C/Villarroel 170, 08036 Barcelona, Spain, Tel.: 3493227 9846, Fax: 349345166 38, e-mail: 30064gsl@comp.es

Received 12-09-06, Revised 22-02-07, Accepted 10-03-07
These two review articles did not specifically address SAH.

The aim of the present commentary is to underscore which aspects of BI related pituitary dysfunction need further research before specific clinical recommendations are formulated and to highlight practical points in the clinical management of these patients after the acute admission phase.

\section{WHO SHOULD BE SCREENED?}

According to the available data, there is consensus that universal screening should be recommended in all patients with moderate-to-severe TBI (Glasgow Coma Scale- GCS- 3 to 12), ${ }^{3,10}$ although patients with severe disability (vegetative states) who will not benefit from hormonal replacement therapy are excluded from non-vital hormone deficiency assessment. In mild cases (GCS $\geq 13$ ), screening is indicated only if there are signs or symptoms of pituitary dysfunction. Similarly, universal screening in all patients with SAH who may potentially benefit from hormones replacement therapy is also recommended.

Universal screening as opposed to selective screening based on clinical characteristics. Here we face the first unresolved question: are there specific symptoms and signs in patients with BI related hypopituitarism? Most prospective studies have not provided systematic clinical data on patients with BI with and

Grant support: supported by a grant from Pfizer Endocrine Care, Spain 
without hormone deficiencies., ${ }^{411-13}$ Agha reported hormonal outcome in 102 consecutive TBI survivors 6 to 36 months after the event, clinical evaluation of patients being carried out via a questionnaire related to the Quality of Life as a measure of growth hormone deficiency (AGHDAQol). ${ }^{11}$ There were no differences in quality of life between $\mathrm{GH}$ deficient and GH sufficient patients. Leal-Cerro selected patients with a custom-made questionnaire; although no data regarding the questionnaire were provided $33 \%$ of the patients showed no signs or symptoms of hypopituitarism according to the questionnaire. Of the remainder, $24.7 \%$ showed some form of hypopituitarism upon testing ${ }^{8}$. To date, the majority of studies have failed to identify clinical predictors of pituitary dysfunction. ${ }^{3,47-9,12}$ However, Bondanelli et al found a relationship between pituitary dysfunction and the severity of the BI evaluated by the GCS, ${ }^{5}$ while Benvenga, in a retrospective review, found an association of the occurrence and the duration of coma with the severity of the BI. ${ }^{1}$

Clinical evaluation of hypopituitarism is difficult because signs and symptoms may be subtle and unspecific and may mimic the neuropsychological sequellae of TBI. ${ }^{10,14}$ Besides, clinical characteristics of mild hypogonadism, hypothyroidism or hypoadrenalism, or mild general hypopituitarism may be difficult to recognize. This is the reason why universal screening with baseline hormonal assessment seems to be the most reasonable approach. ${ }^{3,10}$ Furthermore, efforts should be made in future clinical studies to define clinical characteristics of hypopituitarism in patients with TBI and to identify clinical variables that may predict pituitary dysfunction. Since hypogonadism, hypoadrenalism and hypothyroidism are easier to diagnose than growth hormone deficiency (GHD) with basal testing, it is important to define a clinical protocol to determine which patients will benefit from GHD screening. In the absence of a simple validated questionnaire of hypopituitarism, the AGDAQoL test may be a useful tool, albeit non-specific, to identify low quality of life that may be due to GHD, which is the most prevalent pituitary deficiency 12 months after TBI. ${ }^{4,15}$

In summary, universal hormonal screening should be conducted in all patients with moderate-to-severe TBI, in all patients with SAH and in any degree of
TBI when there is clinical suspicion. Patients in the vegetative state who are unlikely to benefit from hormone therapy are excluded from non-vital hormone assessment. It is advisable that a short clinical scale for hypopituitarism evaluation be defined. The AGHDAQoL may be useful in quality of life evaluation.

\section{HOW SHOULD PATIENTS BE EVALUATED?}

Baseline hormonal testing should be performed in all patients. Screening for hypopituitarism will include tests listed in Table 1 with the following considerations:

\section{A. Pituitary-gonadal axis}

The pituitary-gonadal axis in men is evaluated with $\mathrm{LH}, \mathrm{FSH}$ and testosterone. In women with a history of regular menstrual periods, no assessment is needed; however, in women with menstrual problems or amenorrea, LH, FSH and estradiol have to be measured.

\section{B. Pituitary-adrenal axis}

Pituitary-adrenal evaluation is performed with early morning plasma cortisol measurement. Cut-off values for the diagnosis of adrenal insufficiency are different in the acute admission phase from those in outpatient evaluation. Acute cortisol levels $<200$ $\mathrm{nmol} / \mathrm{L}(7.24 \mu \mathrm{g} / \mathrm{dl})$ suggest ACTH deficiency and replacement therapy is indicated; between 200 and $400 \mathrm{nmol} / \mathrm{L}(14.5 \mu \mathrm{g} / \mathrm{dl})$, clinical judgement should be applied to decide which patients need treatment ${ }^{16}$. After 3-6 months, baseline cortisol under $98 \mathrm{nmol} / \mathrm{L}$ $(3.5 \mu \mathrm{g} / \mathrm{dl})$ is diagnostic of hypoadrenalism, over 285 $\mathrm{nmol} / \mathrm{L}(10 \mu \mathrm{g} / \mathrm{dl})$ rules out adrenal deficiency and between 3.5 and $10 \mu \mathrm{g} / \mathrm{dl}$ requires provocative test-

Table 1. Screening for hypopituitarism 3-6 months after brain injury

\begin{tabular}{ll}
\hline Axis & Measurements \\
\hline Gonadal & $\begin{array}{l}\text { LH* }^{*}, \text { FSH}^{*} \text {, testosterone (men) or estradiol (wom- } \\
\text { en)* }\end{array}$ \\
Adrenal & $\begin{array}{l}\text { Baseline cortisol } \\
\text { ACTH stimulation test when low cortisol (see text) }\end{array}$ \\
Thyroid & TSH, free T4, free T3
\end{tabular}

*(Not needed in eumenorrheic women) 
ing. ${ }^{15,17}$ ACTH stimulation tests (low and standard dose) have been found to be of poor reliability in the diagnosis of adrenal deficiency in patients with baseline cortisol between 3.5 and $10 \mu \mathrm{g} / \mathrm{dl} ;{ }^{18}$ therefore, the Insulin Tolerance test (ITT) will be done when not contraindicated. The CRH test in one study yielded $96 \%$ specificity but poor sensitivity $(76 \%)$, using a cut-off cortisol response of $\leq 377 \mathrm{nmol} / \mathrm{L}$ $(13.6 \mu \mathrm{g} / \mathrm{dl}),{ }^{17}$ and may be considered when the ITT is contraindicated.

\section{Pituitary-thyroid axis}

$\mathrm{TSH}$, free $\mathrm{T} 4$ and free $\mathrm{T} 3$ measurements are required in the assessment of central hypothyroidism; ${ }^{19}$ however, studies have shown that in other causes of central hypothyroidism, including cranial irradiation and pituitary tumors, baseline testing had a poor sensitivity of $73 \% .^{19-21}$ This is an issue that has not been specifically addressed in the context of TBI. Most prospective studies have included only one basal thyroid function measurement. Leal-Cerro did TRH stimulation testing to confirm central hypothyroidism only in those patients who already showed low FT4; therefore, sensitivity of the dynamic test as compared to the basal values cannot be assessed. ${ }^{8}$ Lieberman et al did TRH testing in 27 of 70 adults after TBI. ${ }^{9}$ Of the 15 patients with low FT4 and/or TSH, 6 underwent TRH testing and 3 of them had subnormal TSH response. Clinical scales (Billewicz or that modified by Zulewski) are not sensitive either, but can be useful in monitoring signs and symptoms. ${ }^{22}$ More studies are essential to clarify which is the best way to diagnose hypothyroidism in BI and whether the TRH test should be included in clinical protocols. In the meantime, we propose use of baseline thyroid function tests (TSH, FT4 and FT3) and repetition of the measurement in any case with clinical suspicion of hypothyroidism even in the presence of one set of normal tests.

\section{Growth hormone assessment}

There is no doubt that gonadal, thyroid and adrenal deficiencies should be ruled out and treated. However, whether GHD - in patients with completed linear growth - in the context of BI should be treated and when treatment should be commenced requires more specific studies. This is a crucial issue, since GHD is not easily diagnosed with basal testing. The largest systematic prospective study of pituitary function after $\mathrm{BI}^{4}$ found that GHD is the most common pituitary deficit; 20 and $22 \%$ of patients (TBI and $\mathrm{SAH}$, respectively) had isolated severe GHD at 12 months. To rule out GHD, provocative testing is necessary. ${ }^{23}$ Universal provocative testing in all patients with moderate-to-severe TBI is both troublesome and expensive for the patient. Moreover, since patients with TBI are young, sometimes without previous morbidity, TBI has a major impact in terms of long-term medical care. We have observed that many patients with TBI are reluctant to undergo dynamic blood testing or any further medical evaluation. Before including dynamic testing in routine protocols, clinical consequences of GHD in these patients should be more precisely defined and the effects of GH treatment in this setting need to be proven in randomised clinical trials. Clinical studies are ongoing and their results are needed before specific recommendations are formulated. For the time being, we propose that the measurement of IGF-1 be used to identify GHD (due to its high specificity) and the AGHDAQoL be used to identify which patients may benefit from $\mathrm{GH}$ therapy. Therefore, provocative GH testing, carried out when other hormones have been replaced and at the appropriate time to rule out transitory hormone deficits, may be worthwhile.

The only available data to date regarding $\mathrm{GH}$ treatment in TBI patients are derived from analysis of the KIMS epidemiological study. ${ }^{24}$ In this study, patients with GHD after TBI who received GH replacement therapy were shorter than hypopituitary patients due to other causes, suggesting that GHD management was delayed. Patients in KIMS with TBIinduced pituitary dysfunction usually had 3 additional pituitary deficiencies, which is the least common situation found in recent prospective studies of TBI patients. Data on isolated GHD in TBI patients are consequently lacking and are needed before universal $\mathrm{GH}$ provocative testing is recommended. However, special attention should be paid to TBI in pediatric or adolescent patients who have not completed linear growth. Such patients should be studied and GHD should be ruled out.

Which is the best provocative test to diagnose GHD in TBI patients is another important unresolved issue. The insulin tolerance test (ITT) is the gold standard 
but it is sometimes contraindicated in this setting. Tests using GHRH plus arginine or secretagogues may miss hypothalamic causes of GHD, which may be frequent according to pathology studies. ${ }^{6}$ Therefore, when GH deficiency is suspected and GH treatment is considered, the ITT, when not contraindicated, is the best choice. When the ITT is contraindicated, the glucagon test or tests that use GHRH can be considered, although the former yields some false positive results ( 0 to $16 \%$ according to different studies) $)^{9,25}$ while with GHRH tests a proportion of false negative results are expected.

\section{WHEN SHOULD THE HORMONAL EVALUATION BE PERFORMED?}

Adrenal insufficiency should be evaluated any time it is suspected, including the acute admission phase. ${ }^{2}$ Adrenal and thyroid function should be systematically studied at 3-6 months after the event, or whenever symptoms are present. ${ }^{3,10}$ The gonadal axis can take some time to recover after the stress. There are no specific data on the clinical consequences of delaying gonadal replacement treatment, but taking into account the potential benefit in body composition and exercise capacity, it would be advisable to treat confirmed hypogonadism when it is present at 3-6 months after the episode. Therefore, universal gonadal evaluation is advised at 3-6 months, simultaneously with thyroid and adrenal function evaluation. If these hormonal axes are normal or abnormal, should patients be re-tested at 12 months? According to Agha, ${ }^{11}$ in patients with normal anterior pituitary function at 3-6 months no further assessment is needed. In contrast, Aimaretti et $\mathrm{al}^{12}$ in their prospective study showed that new hormonal deficiencies appeared after 3 months of the BI episode and some deficiencies present at 3-6 months (particularly GH and gonadal deficiencies) proved transitory. Therefore, clinical monitoring of patients with normal results at 3-6 months is advised to detect who may need hormonal testing thereafter. Reassessment of thyroid, adrenal and gonadal function at 12 months should be carried out in patients with abnormal results at 3-6 months to rule out transitory deficiencies. GH deficiency should be evaluated after other pituitary hormone deficiencies are adequately managed. In adult patients, GH evaluation will be performed 12 months after the episode, or whenever symptoms are present and a treatment benefit is expected. In pediatric patients, timing of GHD study will depend on the presence of other pituitary deficiencies and on clinical characteristics. In the case of multiple deficiencies, a GHD study may be done 3-6 months after the episode. If no other pituitary deficiencies are present, growth velocity should be monitored 6-12 months after the BI episode before $\mathrm{GH}$ stimulation tests are performed. Until more data are available, a trial of GH treatment is warranted in cases with proven GHD and symptoms attributable to GHD. There are no data to support further endocrine follow-up beyond 12 months in patients with normal pituitary function since prospective studies have a maximum follow-up of 1 year. However, long-term clinical monitoring of these patients is advised. Based on current findings a new algorithm for endocrine assessment is proposed (Figure 1).

\section{WHO SHOULD EVALUATE PATIENTS FOR POSSIBLE HORMONE DEFICIENCY?}

It is critical that front-line specialists who take care of these patients (neurosurgeons, trauma surgeons and rehabilitation physicians) be aware of the problem; however, given the high prevalence of TBI induced hormonal deficiencies and the non-specificity of clinical signs and symptoms, systematic endocrine referral 3 to 6 months after $\mathrm{BI}$ is recommended. It is advisable to define multidisciplinary cost-effective protocols in which first-line specialists order baseline pituitary function tests and endocrinologists do the clinical evaluation and interpret the hormonal results.

\section{SUMMARY AND CONCLUSIONS}

Although much has been learnt about the endocrine consequences of TBI, many questions still remain before specific practical recommendations may be advanced in terms of evaluation and treatment of such patients. Efforts should be made to define summarized clinical scales to be used for screening and treatment purposes. Universal evaluation with basal hormonal testing is advised in all patients with $\mathrm{SAH}$, in patients with moderate-to-severe TBI and in all cases of TBI with clinical symptoms suggestive of hypopituitarism. Adrenal, gonadal and thyroid func- 


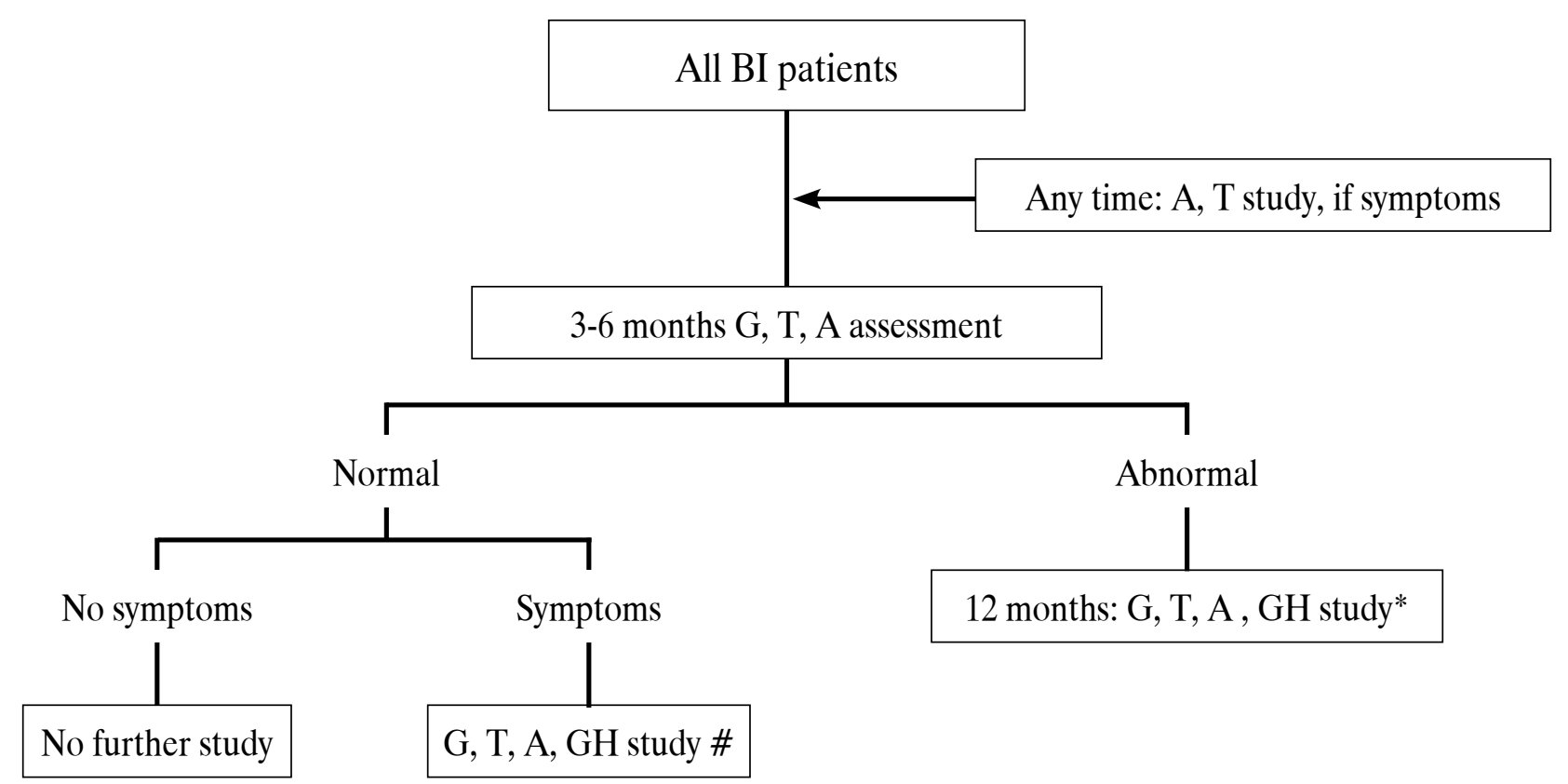

*When other axes normal or adequately managed, \#GH study in adults after 12 months A: adrenal, G: gonadal, T: thyroid

Figure 1. Recommended algorithm for endocrine assessment after brain injury (BI). See text for details.

tion assessment is recommended 3 to 6 months after the episode. Adrenal and thyroid should be studied any time if symptoms are present. Whether thyroid assessment needs provocative testing with TRH needs to be clarified. Reassessment at 12 months should be performed in patients with altered function at 3-6 months and in those with previous normal function but clinical suspicion of hypopituitarism. Provocative testing for GHD may be considered 12 months after the episode, when other pituitary deficiencies are being treated and stable and when a treatment benefit is expected, although scientific evidence of benefit from $\mathrm{GH}$ replacement treatment in this setting is lacking. GHD should be ruled out in all patients with open epiphyses. The AGHDAQoL may help to select adult patients for provocative testing. It is advisable that front-line specialists select patients for study, order baseline pituitary function tests and refer patients to specialised endocrine care for data interpretation and eventual follow-up.

\section{REFERENCES}

1. Benvenga S, Campenni A, Ruggeri RM, Trimarchi F, 2000 Clinical review 113: Hypopituitarism secondary to head trauma. J Clin Endocrinol Metab 85: 1353-1361.

2. Agha A, Rogers B, Mylotte D, et al, 2004 Neuroendocrine dysfunction in the acute phase of traumatic brain injury. Clin Endocrinol (Oxf) 60: 584-591.

3. Agha A, Thompson CJ, 2006 Anterior pituitary dysfunction following traumatic brain injury (TBI). Clin Endocrinol (Oxf) 64: 481-488.

4. Aimaretti G, Ambrosio MR, Di Somma C, et al, 2005 Residual pituitary function after brain injury-induced hypopituitarism: a prospective 12-month study. J Clin Endocrinol Metab 90: 6085-6092.

5. Bondanelli M, De Marinis L, Ambrosio MR, et al, 2004 Occurrence of pituitary dysfunction following traumatic brain injury. J Neurotrauma 21: 685-696.

6. Kornblum RN, Fisher RS, 1969 Pituitary lesions in craniocerebral injuries. Arch Pathol 88: 242-248.

7. Kreitschmann-Andermahr I, Hoff C, Saller B, et al, 2004 Prevalence of pituitary deficiency in patients after aneurysmal subarachnoid hemorrhage. J Clin Endocrinol Metab 89: 4986-4992.

8. Leal-Cerro A, Flores JM, Rincon M, et al, 2005 Prevalence of hypopituitarism and growth hormone deficiency in adults long-term after severe traumatic brain injury. Clin Endocrinol (Oxf) 62: 525-532.

9. Lieberman SA, Oberoi AL, Gilkison CR, Masel BE, Urban RJ, 2001 Prevalence of neuroendocrine dysfunction in patients recovering from traumatic brain injury. J Clin Endocrinol Metab 86: 2752-2756.

10. Ghigo E, Masel B, Aimaretti G, et al, 2005 Consensus 
guidelines on screening for hypopituitarism following traumatic brain injury. Brain Inj 19: 711-724.

11. Agha A, Rogers B, Sherlock M, et al, 2004 Anterior pituitary dysfunction in survivors of traumatic brain injury. J Clin Endocrinol Metab 89: 4929-4936.

12. Aimaretti G, Ambrosio MR, Di Somma C, et al, 2004 Traumatic brain injury and subarachnoid haemorrhage are conditions at high risk for hypopituitarism: screening study at 3 months after the brain injury. Clin Endocrinol (Oxf) 61: 320-326.

13. Tanriverdi F, Senyurek H, Unluhizarci K, Selcuklu A, Casanueva FF, Kelestimur F, 2006 High risk of hypopituitarism after traumatic brain injury: a prospective investigation of anterior pituitary function in the acute phase and 12 months after trauma. J Clin Endocrinol Metab 91: 2105-2111.

14. LaChapelle DL, Finlayson MA, 1998 An evaluation of subjective and objective measures of fatigue in patients with brain injury and healthy controls. Brain Inj 12: 649659.

15. McKenna SP, Doward LC, Alonso J, et al, 1999 The QoLAGHDA: an instrument for the assessment of quality of life in adults with growth hormone deficiency. Qual Life Res 8: 373-383.

16. Agha A, Sherlock M, Thompson CJ, 2005 Post-traumatic hyponatraemia due to acute hypopituitarism. QJM 98: 463-464.

17. Schmidt IL, Lahner H, Mann K, Petersenn S, 2003 Diagnosis of adrenal insufficiency: Evaluation of the corticotropin-releasing hormone test and Basal serum cortisol in comparison to the insulin tolerance test in patients with hypothalamic-pituitary-adrenal disease. $\mathbf{J}$ Clin Endocrinol Metab 88: 4193-4198.

18. Maghnie M, Uga E, Temporini F, et al, 2005 Evaluation of adrenal function in patients with growth hormone deficiency and hypothalamic-pituitary disorders: comparison between insulin-induced hypoglycemia, low-dose ACTH, standard ACTH and CRH stimulation tests. Eur J Endocrinol 152: 735-741.

19. Ferretti E, Persani L, Jaffrain-Rea ML, Giambona S, Tamburrano G, Beck-Peccoz P, 1999 Evaluation of the adequacy of levothyroxine replacement therapy in patients with central hypothyroidism. J Clin Endocrinol Metab 84: 924-929.

20. Rose SR, Lustig RH, Pitukcheewanont P, et al, 1999 Diagnosis of hidden central hypothyroidism in survivors of childhood cancer. J Clin Endocrinol Metab 84: 44724479.

21. Alexopoulou O, Beguin C, De Nayer P, Maiter D, 2004 Clinical and hormonal characteristics of central hypothyroidism at diagnosis and during follow-up in adult patients. Eur J Endocrinol 150: 1-8.

22. Zulewski H, Muller B, Exer P, Miserez AR, Staub JJ, 1997 Estimation of tissue hypothyroidism by a new clinical score: evaluation of patients with various grades of hypothyroidism and controls. J Clin Endocrinol Metab 82: 771-776.

23. Biller BM, Samuels MH, Zagar A, et al, 2002 Sensitivity and specificity of six tests for the diagnosis of adult $\mathrm{GH}$ deficiency. J Clin Endocrinol Metab 87: 2067-2079.

24. Casanueva FF, Leal A, Koltowska-Haggstrom M, Jonsson P, Goth MI, 2005 Traumatic brain injury as a relevant cause of growth hormone deficiency in adults: A KIMSbased study. Arch Phys Med Rehabil 86: 463-468.

25. Gomez JM, Espadero RM, Escobar-Jimenez F, et al, 2002 Growth hormone release after glucagon as a reliable test of growth hormone assessment in adults. Clin Endocrinol (Oxf) 56: 329-334. 